\title{
NUMERICAL SIMULATIONS OF MIXING UNDER SUPERCRITICAL PRESSURES OF A SHEAR COAXIAL INJECTOR USING A HIGH-ORDER METHOD: EFFECT OF OUTER JET TEMPERATURE
}

\section{H. Terashima and M. Koshi}

\author{
University of Tokyo \\ 2-11-16 Yayoi, Bunkyo, Tokyo 113-8656, Japan
}

\begin{abstract}
A three-dimensional (3D) simulation of $\mathrm{N}_{2} / \mathrm{H}_{2}$ mixing for a coaxial injector under a supercritical pressure of $10 \mathrm{MPa}$ is conducted using a highorder numerical method. Two outer $\mathrm{H}_{2}$ jets with injection temperatures of approximately 52 and $462 \mathrm{~K}$ are applied while an inner $\mathrm{N}_{2}$ jet with an injection temperature of approximately $97 \mathrm{~K}$ is applied. The mean and fluctuation properties and instantaneous flow fields are discussed in order to characterize the detailed mixing features for the two injection conditions. A clear dependence of dense-core length on the momentum flux ratio is also demonstrated.
\end{abstract}

\section{INTRODUCTION}

Understanding the mixing and combustion processes under supercritical pressures is of great importance for improving the prediction of rocket combustor performance. For liquid rocket engines, shear coaxial injectors, which provide high performance and good stability with injected oxidizers and fuels, have been commonly used [1]. Thus, several experimental and numerical studies for coaxial injector configurations have been performed in order to understand the complicated mixing and combustion processes under supercritical pressures. However, owing to the extreme pressure and temperature conditions and, therefore, strong thermodynamic variations of these processes, the detailed physics, e.g., the dependence of the mixing characteristics on injection conditions, remain unclear. The present study investigates the mixing of a shear coaxial jet under a supercritical pressure, focusing on the effect of the outer jet temperature.

Mayer and Tamura provided fundamental aspects of the jet mixing of coaxial injectors using flow visualizations and measurements of a liquid oxygen / liquid hydrogen $\left(\mathrm{LOx} / \mathrm{LH}_{2}\right)$ system [2]. They revealed that, with increasing cham- 
ber pressure, the jet surface shows fine thread- or stringy-like structures that rapidly dissolve, and the jet develops nonaxisymmetric snake-like oscillations. Oschwald et al. experimentally investigated coaxial liquid nitrogen / gaseous hydrogen $\left(\mathrm{LN}_{2} / \mathrm{GH}_{2}\right)$ injection at supercritical pressure using spontaneous Raman scattering, providing radial density profiles of $\mathrm{N}_{2}$ and $\mathrm{H}_{2}$ as a function of the location downstream the injector for three injection conditions [3]. Davis and Chehroudi conducted an experimental investigation on a coaxial nitrogen jet under sub- through supercritical pressures [4]. The dense-core length fluctuation was measured using high-speed digital images, and they found a good correlation between the dense-core length and the outer jet to inner jet momentum ratio, which is the useful benchmark data for representing coaxial jet characteristics. Locke et al. [5] visualized the LOx core injected from a coaxial injector of LOx / gaseous oxygen (GOx) using high-speed movies with backlighting. They reported that there is no large oscillatory behavior of the dense core in cold test cases, i. e., the dense-core structures are less disconnected, which is very different from hot-fire test cases. They also made a comparison of dense-core length with a number of earlier correlations, including the data from Davis and Chehroudi [4]. Although experimental studies have provided highly prominent aspects and correlations for characterizing the mixing of coaxial jets, there are still several difficulties with experiments associated with extreme pressure and temperature conditions and with obtaining optical access. Thus, the detection of small-scale structures or highly unsteady flow features is not easily achieved.

Along with experimental studies, computational efforts have been also dedicated to obtaining a detailed understanding of the mixing characteristics of coaxial jets under supercritical pressures. Generally, large-eddy simulation (LES) techniques are used because of its superior performance as compared to the Reynolds-averaged Navier-Stokes (RANS) approach in resolving highly unsteady flow fields. However, the number of 3D LES or direct numerical simulation (DNS) studies for supercritical mixing is still very small. For a single round jet, LES was performed by Zong and Yang, extensively analyzing various effects such as density stratification and property variation on the evolution of cryogenic jets and mixing layers [6]. Schmitt et al. [7] investigated the jet spreading angle and pseudosimilarity behavior based on a comparison with the experiment by Mayer et al. [8], who also reported the stabilizing effect due to the large density gradient between a cryogenic jet and chamber fluid under supercritical pressures. Park [9] reported the effects of turbulence models and subgrid scale (SGS) models on the structures of supercritical nitrogen jets, showing the superiority of LES to RANS. Terashima and Koshi [10] simulated a cryogenic nitrogen jet using a high-order numerical method, showing its unique temperature profile and the physics behind it.

For coaxial jets, mixing studies under supercritical pressures are further limited. Nevertheless, Hosangadi et al. applied a hybrid RANS/LES method to a supercritical $\mathrm{LN}_{2}$ jet for a shear coaxial injector configuration [11]. They found 
that unsteady mixing is dominated by $3 \mathrm{D}$ helical instabilities (which is different from a gas jet with axisymmetric instabilities) and relatively small temperature fluctuations lead to large density fluctuations due to the strong thermodynamic gradients in the transcritical regime. Masquelet et al. [12] made a comparison between experimental results [5] and those obtained using LES, and reasonable agreement was found in experimental time-averaged statistics and distribution of LOx core lengths. Schmitt et al. [13] investigated the structure of transand supercritical nitrogen jets formed by a coaxial injector operating under high pressure using LES and compared their theoretical results with the experimental ones. They showed that when the momentum flux ratio exceeds a critical value, the inner jet is abruptly terminated, which is linked to the formation of a recirculation region near the injection section.

Based on these earlier experimental and computational studies, the present study numerically investigated the high-pressure jet mixing injected from a shear coaxial injector. The primary goal was to investigate the effect of the outer jet temperature on the mixing dynamics. It is well known that in a $\mathrm{LOx} / \mathrm{H}_{2}$ rocket engine system, the temperature of the outer $\mathrm{H}_{2}$ jet is indicative of the onset of instability in the engine $[1,4]$. In this study, the investigation is limited to nonreactive flow fields in order to focus on the mixing characteristics, whereas an injection with colder hydrogen may be significantly affected by the presence of combustion physics. Two outer $\mathrm{H}_{2}$ jets with a cold-jet injection temperature of $52 \mathrm{~K}$ and a warm-jet injection temperature of $462 \mathrm{~K}$ were used while an inner $\mathrm{N}_{2}$ jet with an injection temperature of $97 \mathrm{~K}$ was applied. The mixture ratio and injector dimensions were fixed between the two conditions; thus, the velocity and momentum ratio were different. A high-order numerical method using a sixthorder compact scheme, which was developed by the authors of [14], was applied to investigate the flow fields.

\section{NUMERICAL METHOD}

\subsection{Governing Equations}

The governing equations are the Navier-Stokes equations with the species-mass equations in generalized curvilinear coordinates. The Soave-Redlich-Kwong (SRK) equation of state [15] is used to represent the behavior of cryogenic fluids under supercritical pressures. The present method is constructed so that possible spurious pressure and velocity oscillations due to the peculiarity of the equation of state can be prevented by satisfying the velocity and pressure equilibriums at inviscid interfaces [14]. Thus, the governing equations solved in this study are

$$
\begin{gathered}
\frac{\partial \rho}{\partial t}+\nabla \cdot(\rho \boldsymbol{u})=\nabla \cdot\left(\psi_{\rho} \nabla \rho\right) ; \\
\frac{\partial \rho \boldsymbol{u}}{\partial t}+\nabla \cdot(\rho \boldsymbol{u} \otimes \boldsymbol{u}+p \boldsymbol{\delta}-\boldsymbol{\tau})=\nabla \cdot\left(\psi_{\rho}(\boldsymbol{u} \otimes \boldsymbol{g}) \nabla \rho\right) ;
\end{gathered}
$$




$$
\begin{gathered}
\frac{\partial p}{\partial t}+\boldsymbol{u} \cdot \nabla p=-\rho c^{2} \nabla \cdot \boldsymbol{u}+\frac{\alpha_{p}}{c_{v} \beta_{T}}\left[\frac{1}{\rho}(\boldsymbol{\tau}:(\nabla \otimes \boldsymbol{u})-\nabla \cdot \boldsymbol{q})\right] \\
\frac{\partial \rho Y_{i}}{\partial t}+\nabla \cdot\left(\rho \boldsymbol{u} Y_{i}\right)-\nabla \cdot\left(\rho D_{i} \nabla Y_{i}\right)=\nabla \cdot\left(\psi_{\rho} \nabla \rho Y_{i}\right) .
\end{gathered}
$$

Here, the pressure evolution Eq. (3) is used instead of the total energy conservation equation to maintain the pressure equilibrium. The right-hand side terms in Eqs. (1), (2), and (4) are the consistent artificial diffusion terms; the artificial diffusion term for the mass equation serves to capture interfaces with large density contrasts, the one for the momentum equation is introduced to satisfy the velocity equilibrium, and the one for the species-mass equations is introduced to satisfy the relation of $\sum_{i} Y_{i}=1$. In Eqs. (1)-(4), $\rho$ is the density; $\boldsymbol{u}$ is the velocity vector; $p$ is the pressure; $\boldsymbol{\tau}$ is the stress tensor; $c$ is the speed of sound; $\boldsymbol{q}$ is the heat flux vector; $Y_{i}$ is the mass fraction of species $i ; D_{i}$ is the diffusion coefficient of species $i ; c_{v}=(\partial e / \partial T)_{V}$ is the heat capacity at constant volume with $e$ being the internal energy, $T$ being the temperature, $V=M_{m} / \rho$ being the specific volume, and $M_{m}$ being the molar weight of the mixture; $\alpha_{p}=(\partial V / \partial T)_{p} / V$ is the thermal expansion coefficient; and $\beta_{T}=-(\partial V / \partial p)_{T} / V$ is the isothermal compressibility coefficient. The coefficient $\psi_{\rho}$ is given below and $\boldsymbol{g}=\left(\begin{array}{lll}1 & 1 & 1\end{array}\right)$ is a row vector. The dynamic viscosity and the thermal conductivity are calculated by the model of Chung et al. [16] and the physical diffusion coefficient is calculated using the model of Riazi and Whitson [17]; no SGS models are used, i. e., it is considered that the dissipation from the artificial diffusion terms added in Eqs. (1), (2), and (4) can be regarded as numerical dissipation to model unresolved small scales in the same manner as an explicit SGS model.

The SRK model [15] is written as

$$
p=\frac{R_{u} T}{V-b}-\frac{a(T)}{V^{2}+b V} .
$$

Here, $R_{u}$ is the universal gas constant and the effective volume of the molecules; $b$ is calculated as $b=\sum_{i} \chi_{i} b_{i}$ where $\chi_{i}$ is the mole fraction and $b_{i}$ $=0.0866 R_{u} T_{\mathrm{cr}, i} / p_{\mathrm{cr}, i}$, the subscript $\mathrm{cr}$ denotes the critical point. The intermolecular interaction is given as

$$
a(T)=\sum_{i} \sum_{j} \chi_{i} \chi_{j}\left(a_{i} \alpha_{i}(T)\right)^{1 / 2}\left(a_{j} \alpha_{j}(T)\right)^{1 / 2} .
$$

Here, $a_{i}=0.4275 R_{u}^{2} T_{\mathrm{cr}, i}^{2} / p_{\mathrm{cr}, i}$ and the function $\alpha_{i}(T)$ is

$$
\alpha_{i}(T)=\left[1+f\left(\omega_{i}\right)\left(1-\left(\frac{T}{T_{\mathrm{cr}, i}}\right)^{1 / 2}\right)\right]^{2}
$$

where $f\left(\omega_{i}\right)=0.480+1.574 \omega_{i}-0.176 \omega_{i}^{2}$ in which $\omega_{i}$ is the acentric factor of species $i$. 


\subsection{Artificial Diffusions}

The artificial diffusion coefficient, $\psi_{\rho}$, in Eqs. (1), (2), and (4) is modeled by extending the idea of the localized artificial diffusivity (LAD) method [18-20] as

$$
\psi_{\rho}=C_{\rho} \overline{\frac{c}{\rho}\left|\sum_{l=1}^{n_{d}} \frac{\partial^{r} \rho}{\partial \xi_{l}^{r}} \Delta \xi_{r}^{l} \Delta_{l}\right|}
$$

where $n_{d}=1 \ldots 3$ is the number of dimensions; $\xi_{l}$ refers to the generalized curvilinear coordinates $\xi, \eta$, and $\zeta$ when $l$ is 1,2 , and $3 ; \Delta \xi_{l}$ is the grid spacing in generalized curvilinear coordinates usually set to 1 ; and $\Delta_{l}$ also corresponds to the grid spacing in physical space, i. e., $\Delta x, \Delta y$, and $\Delta z$ when $l$ is 1,2 , and 3. The overbar denotes an approximate truncated Gaussian filter [18]. The order of the derivative $r$ is taken to be 4 and the derivative term is evaluated by an explicit fourth-order central differencing scheme [21]. $C_{\rho}$ is an adjustable user-specified constant; $C_{\rho}=0.02$ is used in this study based on a previous study [14].

Further, as in previous studies [19,20], to keep the mass fraction between 0 and 1 , an artificial diffusion coefficient is added to the diffusion coefficient, $D_{i}$. Thus, the diffusion coefficient is evaluated as

$$
D_{i}=D_{f, i}+D_{i}^{*}
$$

where the subscript $f$ and superscript asterisk denote the physical and artificial fluid properties, respectively. In this study, the artificial diffusion coefficient, $D_{i}^{*}$, is modeled as

$$
D_{i}^{*}=C_{Y} \overline{c\left[\left(Y_{i}-1\right) H\left(Y_{i}-1\right)-Y_{i}\left(1-H\left(Y_{i}\right)\right)\right] \Delta_{Y}}
$$

where $C_{Y}=50$ is used [14]; $\Delta_{Y}=(\Delta x \Delta y \Delta z)^{1 / 3}$ is the local grid spacing [19]; and $H$ is the Heaviside function. The overbar denotes the approximate truncated Gaussian filter as in Eq. (5). This term was found to be helpful in reducing over/undershoots of the mass fraction, thus suppressing temperature oscillations [14].

\subsection{Numerical Schemes}

The spatial derivatives are evaluated using a sixth-order compact differencing scheme [21]. A third-order total variation diminishing Runge-Kutta scheme [22] is used for temporal integration. Eighth-order low-pass filtering $[21,23]$ is applied once in each direction to the conservative variables $\rho, \rho u$, and $\rho Y_{i}$ and the pressure $p$ after the final stage of the Runge-Kutta scheme. A free parameter in the filtering scheme, $\alpha_{f}$, is fixed at $\alpha_{f}=0.495$. 


\section{RESULTS AND DISCUSSIONS}

\subsection{Conditions}

A schematic of the coaxial injector used in this study is shown in Fig. 1. Although various injector dimensions have been considered, the present study adopts an inner diameter of $d_{1}=2.0 \mathrm{~mm}$, an outer diameter of $d_{2}=4.0 \mathrm{~mm}$, and a post-tip thickness of $l_{1}=0.36 \mathrm{~mm}$ as a baseline model, following similar dimensions of a shear-coaxial injector element used in an earlier experiment [5]. A rectangular computational domain of $100 d_{1} \times 12.8 d_{1} \times 12.8 d_{1}$ is used to represent a combustion chamber, also following the dimensions of the experimental cross-sectional area [5], whereas the downstream length is taken to be somewhat shorter for brevity. The present study assumes that cryogenic nitrogen from the inner tube and hydrogen from the outer tube were injected to simulate the mixing process between oxidizer and fuel in combustion chambers. The pressure in the chamber is set to a supercritical pressure of $10.0 \mathrm{MPa}$. Table 1 presents the injector flow conditions used where the velocity is selected so that the injector mixture ratio is close to a typical value of 5.0-6.0 in liquid rocket engines $[2,5]$. To investigate the effects of outer jet temperature on mixing behaviors, two injector flow conditions (Cases 1 and 2) are considered under a constant mixture ratio. Note that the momentum and velocity ratios are different between the two cases.

In addition to the two coaxial jet cases, a single nitrogen jet is simulated for comparison (i. e., no outer hydrogen jet is injected; hereafter, this condition is called Case 3). Note that the temperature values in Table 1 are estimated with the SRK equation of state. Here, Case 1 is considered as a reference condition,
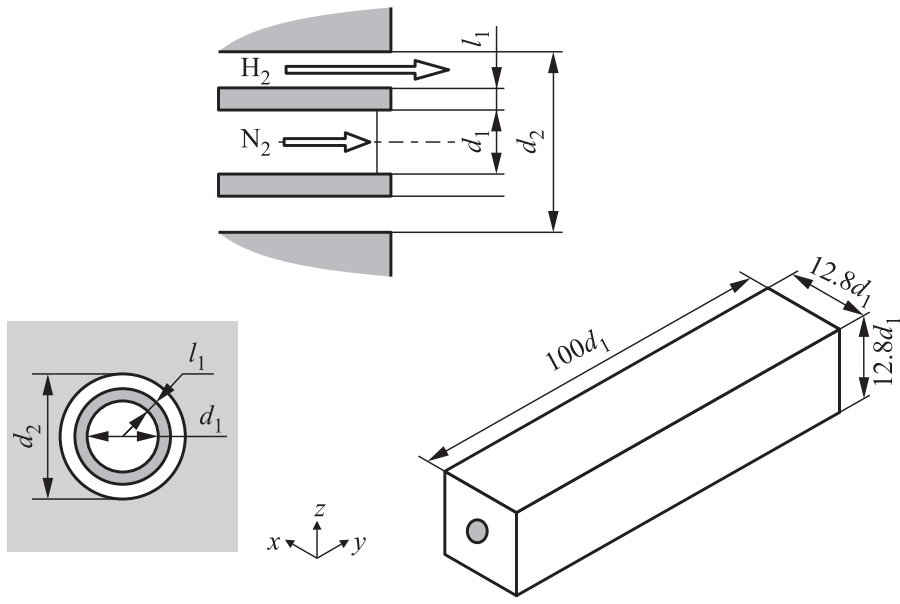

Figure 1 Schematic of injector dimensions and computational domain (not to scale) 
Table 1 Computational conditions, where the pressure is $10 \mathrm{MPa}$. The subscripts $i$ and $o$ mean the quantities of the inner and outer jets, respectively

\begin{tabular}{|c|c|c|c|c|c|c|}
\hline & $\begin{array}{c}\rho, \\
\mathrm{kg} / \mathrm{m}^{3}\end{array}$ & $\begin{array}{l}u, \\
\mathrm{~m} / \mathrm{s}\end{array}$ & $T, \mathrm{~K}$ & $\mathrm{Re} \cdot 10^{5}$ & $\mathrm{MR}=\frac{(\rho u A)_{\mathrm{N}_{2}}}{(\rho u A)_{\mathrm{H}_{2}}}$ & $J=\frac{(\rho u u)_{o}}{(\rho u u)_{i}}$ \\
\hline Inner jet, $\mathrm{N}_{2}$ & 750 & 15 & 96.62 & 1.48 & & \\
\hline Chamber, $\mathrm{N}_{2}$ & 40 & 0 & 810.4 & & & \\
\hline Case 1 , outer jet, $\mathrm{H}_{2}$ & 5 & 200 & 461.7 & 1.65 & 5.23 & 1.185 \\
\hline Case 2 , outer jet, $\mathrm{H}_{2}$ & 50 & 20 & 51.69 & 2.98 & 5.23 & 0.1185 \\
\hline
\end{tabular}

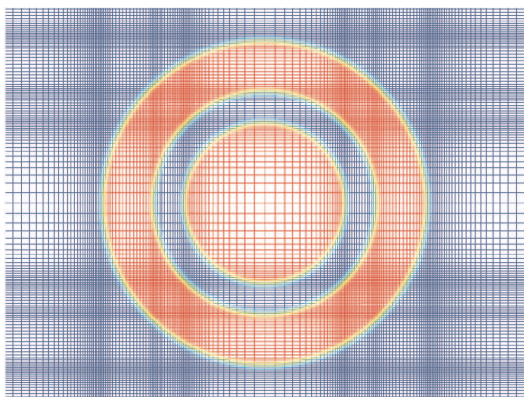

(a)

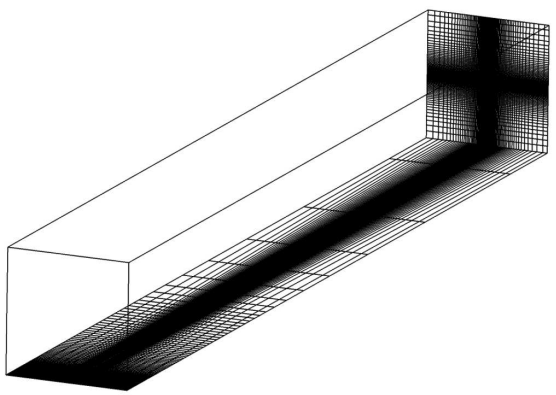

(b)

Figure 2 Computational grid $(245 \times 201 \times 201)$ : $(a)$ close-up view on $y-z$ plane (the distribution of an error function is plotted, where the red region corresponds to the jet inlets and the blue to the wall (face plate)) and $(b)$ the whole view

whereas Case 2 presents the effect of outer jet temperature under the same mixture ratio, and Case 3 is used to investigate the effect of the outer jet.

Figure 2 shows the computational grid used in this study. The grid consists of $245 \times 201 \times 201$ grid points, in which the minimum grid spacing in the $y$ and $z$ directions is $\Delta s=d_{1} / 100=20 \mu \mathrm{m}$ and the one in the streamwise $(x)$ direction is $\Delta s=3 d_{1} / 100=60 \mu \mathrm{m}$. As shown in Fig. $2 a$, the grid is clustered near the shear layers generated by the inner and outer jets. Based on the authors' previous study for a single-species round jet simulation [10], one may expect that the grid used here would be sufficient for resolving coaxial jet dynamics such as mean and fluctuation characteristics.

The profile of the primitive variables $\boldsymbol{q}=\left(\rho, u, v, w, p, Y_{i}\right)$ at the inlet is smoothly generated in the radial direction using an error function, $\operatorname{erf}\left(\Delta d /\left(C_{\epsilon} \Delta s\right)\right)$, as in [24], where $\Delta d$ is the distance from the center and $C_{\epsilon}$ is the adjustable parameter: $C_{\epsilon}=3.0$ is used here. As an example, the profile of an error function on $x=y=0$ is shown in Fig. 3 (a spatial distribution on the 


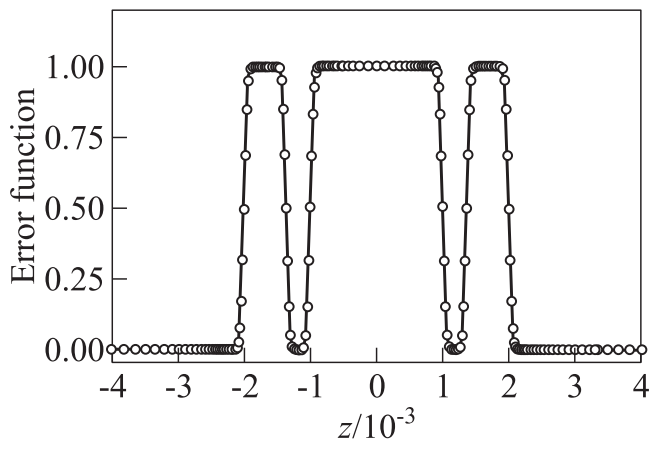

Figure 3 An example of the profile of an error function on $x=y=0.0 ; C_{\epsilon}=3$ $y-z$ plane is presented in Fig. 2a). No perturbations are introduced; since strong unsteady flow fields are generated owing to the shear layers between the two jets, considerable influence of such perturbation or fluctuation on jet dynamics would not be expected [11]. A slip wall boundary is imposed at the boundaries in the $y$ - and $z$-directions. At the outlet, the pressure is fixed to a chamber pressure of $10 \mathrm{MPa}$ and the other variables are extrapolated. The Courant-Friedrichs-

Lewy (CFL) number is 0.4 , which corresponds to a time step size of approximately $\Delta t=4.4 \cdot 10^{-3} \mu \mathrm{s}$ for Case 1 and approximately $\Delta t=9 \cdot 10^{-3} \mu \mathrm{s}$ for Case 2. The difference of $\Delta t$ comes from the speed of sound of the outer $\mathrm{H}_{2}$ jet. The time step size in Case 3 is thus somewhat larger, approximately $1.3 \cdot 10^{-2} \mu \mathrm{s}$. For Case 1, the statistical values are estimated using the data for approximately $0.65 \mathrm{~ms}$ after a 3.0 -millisecond transient stage, which means that the number of flow-throughs for the averaging is almost 3.5 based on the reference convective time, $20 d_{1} /\left(0.5\left(u_{i}+u_{o}\right)\right)$ where the subscripts $i$ and $o$ denote the inner and outer jets, respectively. For Case 2, the number of flow-throughs is almost 3.4 using the data for $7.8 \mathrm{~ms}$ after a 2.8 -millisecond transient stage.

\subsection{Instantaneous Flow Fields}

Figure $4 a$ shows the instantaneous distributions of the logarithmic scale of density for Cases 1 and 2, which are used for highlighting the density differences. Apparently, for both cases, highly unsteady flow fields appear owing to the shear forces provided by the interaction between the inner $\mathrm{N}_{2}$ and the outer $\mathrm{H}_{2}$ jets. The logarithmic scale of density, which may correspond to the backlight image of experiments, qualitatively shows that the dense core of the inner $\mathrm{N}_{2}$ jet in Case 1 disappears faster than that in Case 2, indicating that the outer $\mathrm{H}_{2}$ jet of Case 1 effectively enhances mixing with the inner $\mathrm{N}_{2}$ jet. On the other hand, due to the small velocity of the outer $\mathrm{H}_{2}$ jet and, therefore, a quite small momentum ratio of approximately 0.12 , the outer jet in Case 2 is not sufficient for breaking up the dense core of the inner $\mathrm{N}_{2}$ jet.

A qualitative comparison of the mass fraction of $\mathrm{H}_{2}$ is presented in Fig. $4 b$. It is shown that the mass fraction of the $\mathrm{H}_{2}$ jet of Case 1 decays rapidly owing to the strong shear and the distribution is widely diffused across the entire downstream 

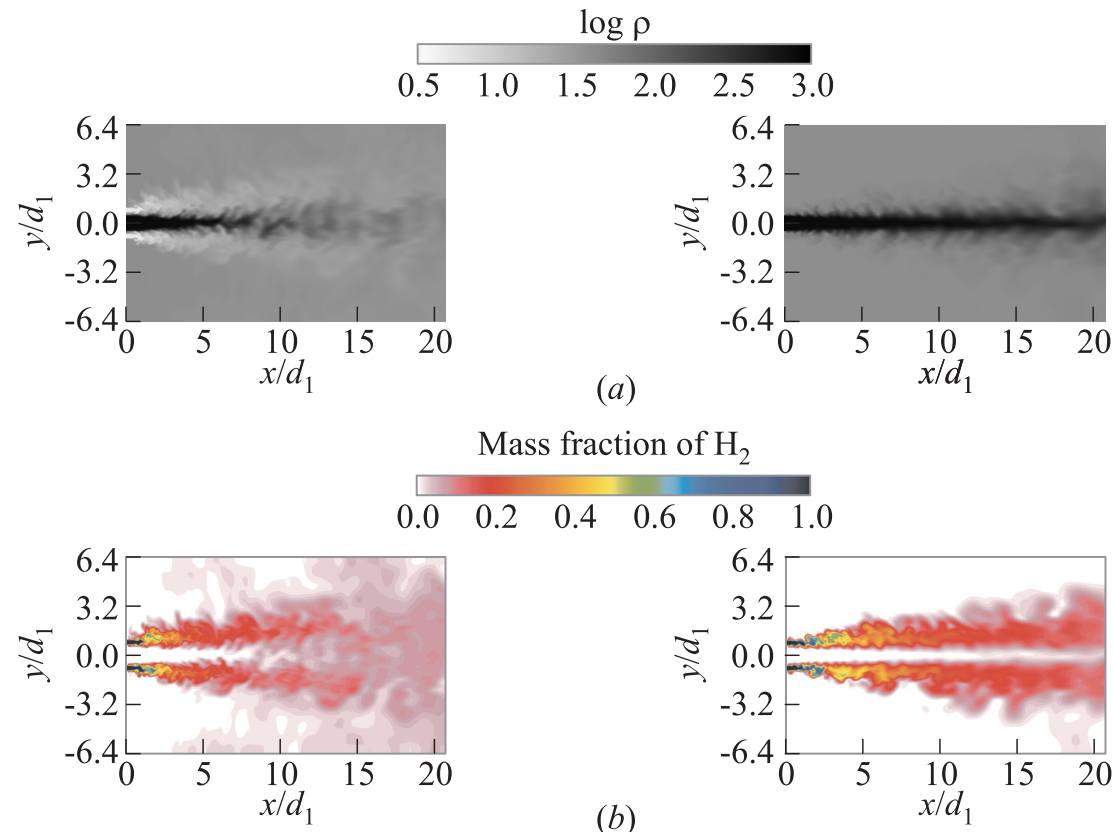

Mass fraction of $\mathrm{H}_{2}$

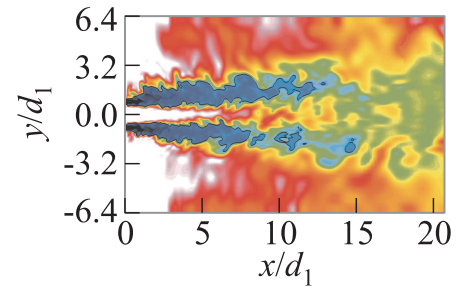

Temperature / K

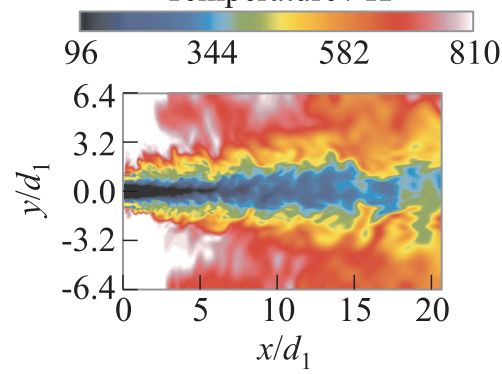

(b)

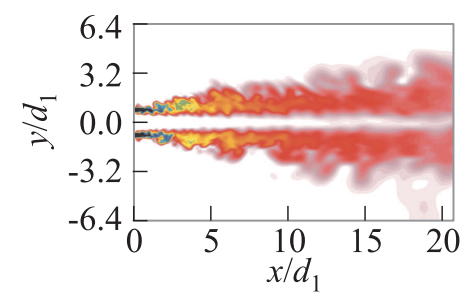

(c)

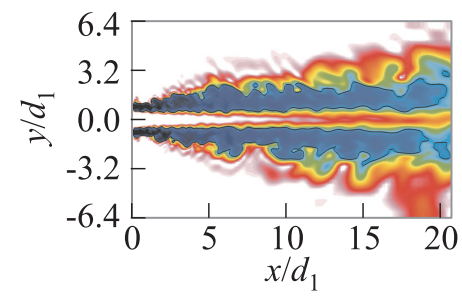

Temperature / K

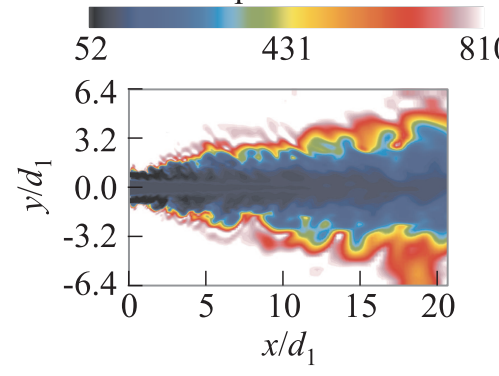

Figure 4 Instantaneous distributions for Cases 1 (left column) and 2 (right column): (a) logarithmic scale of density; (b) mass fraction of $\mathrm{H}_{2}$; $(c)$ mole fraction of $\mathrm{H}_{2}$ and a contour of mole fraction $X_{\mathrm{H}_{2}}=2 / 3$; and $(d)$ temperature 
region. In Case 2, although the mixing behavior near the inlet is similar to that of Case 1, the spreading angle is more limited, generating a confined region of the mass fraction of $\mathrm{H}_{2}$.

Figure $4 c$ shows the instantaneous distribution of the mole fraction of $\mathrm{H}_{2}$, $X_{\mathrm{H}_{2}}$, and the contour of $X_{\mathrm{H}_{2}}=2 / 3$, which corresponds to the equilibrium ratio of 1.0 if $\mathrm{N}_{2}$ is replaced with $\mathrm{O}_{2}$. It is found that in Case 2, a rich $\mathrm{H}_{2}$ region extends to the downstream region of approximately $x / d_{1}=20$ since the mixing is not as thorough, whereas such a rich $\mathrm{H}_{2}$ region as in Case 1 disappears at approximately $x / d_{1}=10$. Thus, based on this result, a possible scenario may be that an injection condition such as Case 2, i. e., low momentum flux or low velocity ratio, leads to the presence of propellant in the downstream region, potentially inducing combustion after a time delay. However, mixing with such colder hydrogen may be significantly affected by the presence of combustion, e. g., the effects of chemical reactions or flammability limits.

The temperature distribution shown in Fig. $4 d$ presents almost the same features between two cases, the change in temperature of the inner $\mathrm{N}_{2}$ jet is minimal,

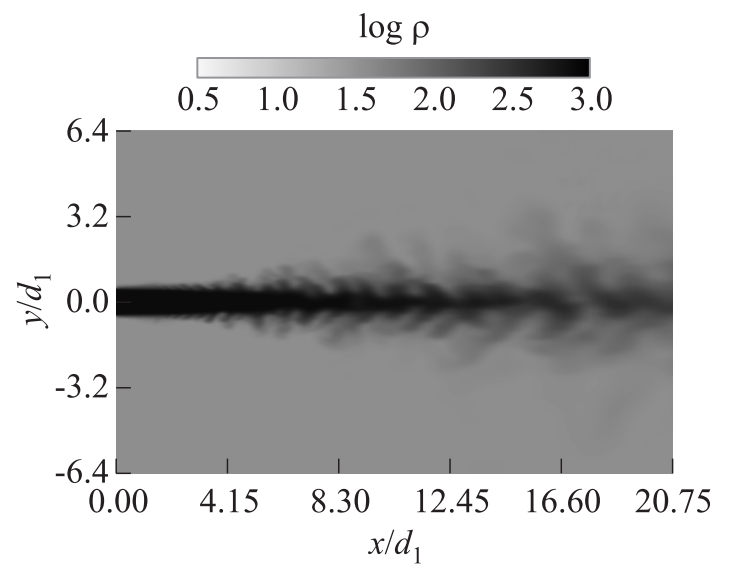

Figure 5 Instantaneous distributions of logarithmic scale of density for a single $\mathrm{N}_{2}$ jet even in the downstream region, and the jet spreading angle is also limited. The present results demonstrate that even if the same mixture ratio is set for a coaxial injector, the resultant mixing fields are considerably different, depending on outer jet conditions. Low-temperature injection of the outer jet and, therefore, low momentum flux ratio provide less mixing for coaxial injectors.

A single-jet simulation (Case 3) was also carried out in order to investigate the effect of the outer jet on mixing characteristics. Figure 5 shows the instantaneous distributions of the logarithmic density for the single-jet case. As compared to Case 2 shown in Fig. $4 a$, the behavior of jet surface looks similar, whereas a delay of instability on the jet surface is recognized because of the absence of outer jet. However, the dense core of the single jet seems to eventually disappear faster and spread wider than that in Case 2, indicating that the outer jet in Case 2 unexpectedly stabilizes the inner jet. This stabilization in Case 2 may come from the lower velocity difference of $\Delta u=u_{i}-u_{o}=-5 \mathrm{~m} / \mathrm{s}$ between the inner and outer jets as compared to the velocity difference of $\Delta u=u_{i}-u_{\infty}=15 \mathrm{~m} / \mathrm{s}$ in the single jet. 


\subsection{Mean Flow Characteristics}

Figure $6 a$ shows the mean distributions of the logarithmic density for Cases 1 and 2. As discussed in the previous section, a much longer dense core is clearly confirmed in Case 2 also in the mean flow field. The outer $\mathrm{H}_{2}$ jet of Case 2, thus, does not effectively break up the inner $\mathrm{N}_{2}$ jet and, therefore, the mixing is little enhanced. As shown in Fig. $6 b$, there is a large difference in the $x$-velocity field between Cases 1 and 2. In Case 2, the inner $\mathrm{N}_{2}$ jet merges with the outer $\mathrm{H}_{2}$ jet owing to the similar $x$-velocity values, as if one unified jet is created. Seemingly, the $x$-velocity of the unified jet changes little, even downstream.

Figure 7 shows the mean density distribution for the single-jet case (Case 3). Although the dense core in the single-jet case is qualitatively similar to that for Case 2 in terms of the length and the spreading angle, the decay of the dense core in Case 3 looks somewhat faster and the spreading somewhat wider than in Case 2, which are the same features as the instantaneous flow fields.

To make a quantitative comparison of the dense-core length of the inner $\mathrm{N}_{2}$ jet, nondimensional density and temperature profiles on the centerline of the inner $\mathrm{N}_{2}$ jet are shown in Fig. 8. The nondimensional quantity, $f^{*}$, is defined as

$$
f^{*}=\frac{f-f_{\infty}}{f_{i}-f_{\infty}}
$$

where the quantity $f$ corresponds to the density or temperature, and the subscripts $\infty$ and $i$ denote the quantity of the chamber fluid and inner jet, respectively. Following the discussion in [12], it is expected that the values in the range of $0.72<\rho^{*}<0.86$ are considered as the reference values for determining dense-core length because densities between 550 and $650 \mathrm{~kg} / \mathrm{m}^{3}$ correspond to the transcritical transition between liquid and supercritical fluid under $10 \mathrm{MPa}$.

As discussed above for Case 1, the nondimensional density rapidly decreases, reaching the chamber density at approximately $x / d_{1}=15.0$ early; thus, a shorter dense core appears. On the other hand, Case 2 generates a relatively long dense core on the centerline. Even at $x / d_{1}=15$ where the dense core of Case 1 completely disappears, a strong dense-core region still remains. Case 3 (the single-jet case) interestingly generates a shorter dense-core length as compared to Case 2, indicating some stabilization effect of the outer jet on the inner jet. This may be because the velocity shear between the inner $\mathrm{N}_{2}$ jet and the stationary chamber fluid in Case 3 is larger than that between the inner and outer jets in Case 2. Thus, it is both quantitatively and qualitatively indicated that the outer $\mathrm{H}_{2}$ jet injection in Case 2 tends to stabilize the shear on the inner $\mathrm{N}_{2}$ jet surface rather than enhance the mixing between the inner and outer jets.

Along with the density profiles, the nondimensional temperature profiles are shown in Fig. 8b. Although the temperature in Case 1 decreases rapidly, Case 2 


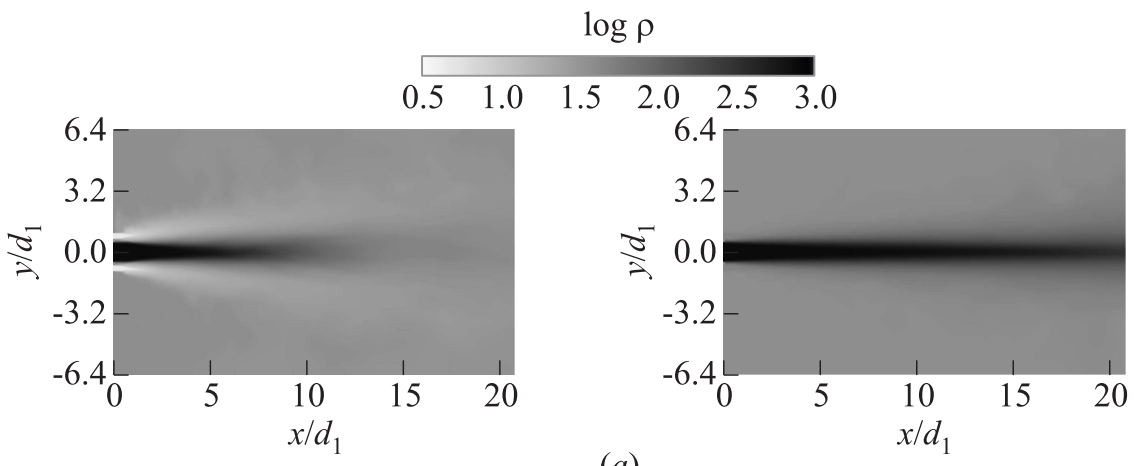

(a)
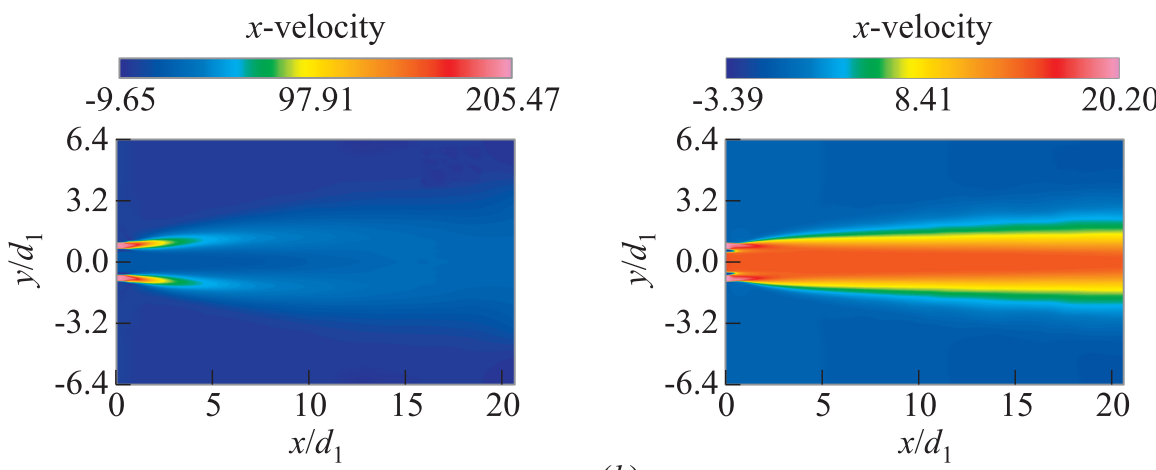

(b)

Temperature / K

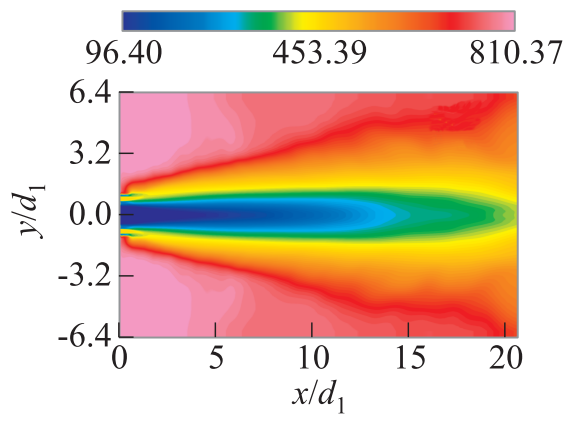

Temperature / K

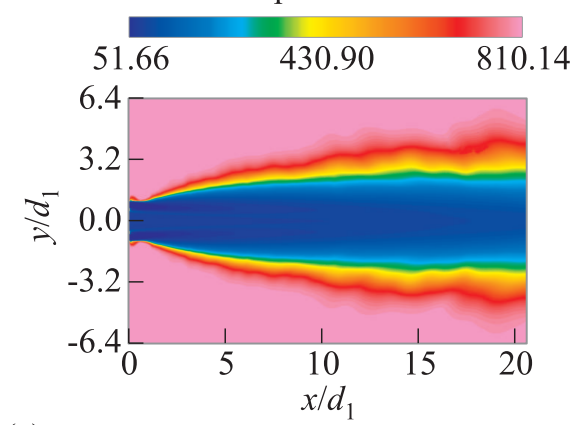

(c)

Figure 6 Mean distributions for Cases 1 (left column) and 2 (right column): (a) logarithmic scale of density; $(b) x$-velocity; and (c) temperature 
shows that the change in temperature on the centerline is minimal even at approximately $x / d_{2}=25$. This is because, in addition to the thermodynamic characteristics of $\mathrm{N}_{2}$, the inner $\mathrm{N}_{2}$ jet is not well mixed with the surrounding $\mathrm{H}_{2}$ jet and chamber $\mathrm{N}_{2}$ fluid, i. e., the inner $\mathrm{N}_{2}$ jet almost follows the static thermodynamic variations. Figure $9 a$ shows a static thermodynamic variation of $\mathrm{N}_{2}$, which represents the small variation of temperature against the density in the range

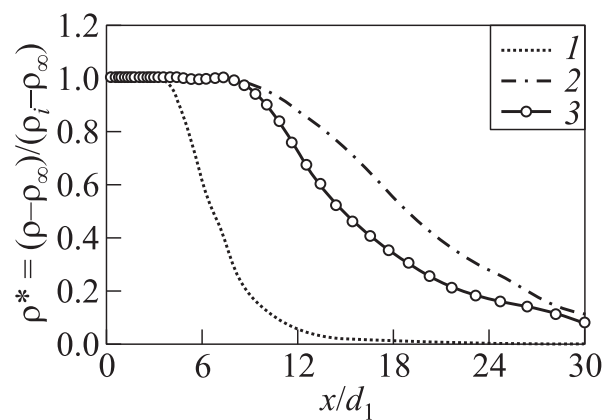

(a)

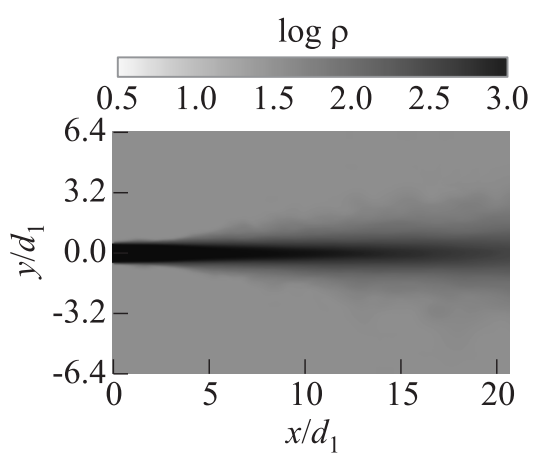

Figure 7 Mean density distribution for a single-jet case (Case 3)

Figure 8 Comparison of mean profiles on the centerline of the inner $\mathrm{N}_{2}$ jet, where the subscripts $\infty$ and $i$ denote chamber fluid and inner jet, respectively: 1 - Case 1 : coaxial jet, 2 - Case 2: coaxial jet, 3 - Case 3 : single jet

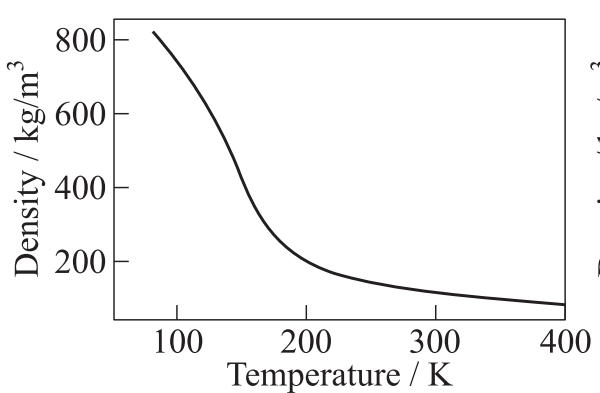

(a)

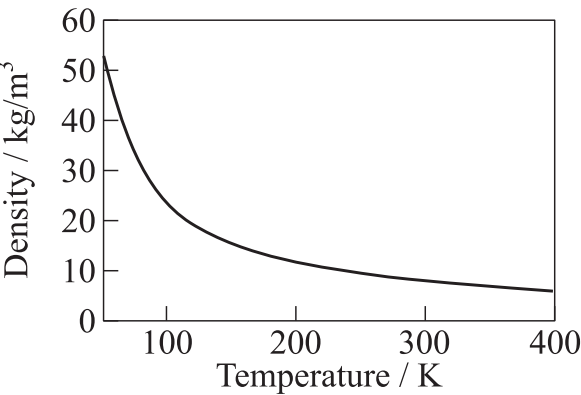

(b)

Figure 9 A static thermodynamic relation of $\mathrm{N}_{2}(a)$ and $\mathrm{H}_{2}(b)$ at $10 \mathrm{MPa}$ from NIST [25] - density as a function of temperature 
of the transcritical transition (100 through $170 \mathrm{~K}$ ). Thus, it is confirmed that even if the density changes, as in Fig. $8 a$, the temperature variation in that range is limited, resulting in the small temperature increase of the inner $\mathrm{N}_{2}$ jet on the centerline.

As for the single-jet case (Case 3), since the single jet is somewhat well mixed with the surrounding chamber fluid as compared to Case 2 (see Fig. 8a), the variation of temperature on the centerline is more pronounced.

\subsection{Fluctuation Flow Characteristics}

Figures $10 a$ and $10 b$ show the distributions of nondimensional fluctuation density, $\rho^{\prime} \rho^{\prime}$, and temperature, $T^{\prime} T^{\prime}$, scaled by the mean distributions, respectively. In Case 1, the interaction between the inner and outer jets generates large density fluctuations, whereas the density fluctuation on the outer jet itself (which
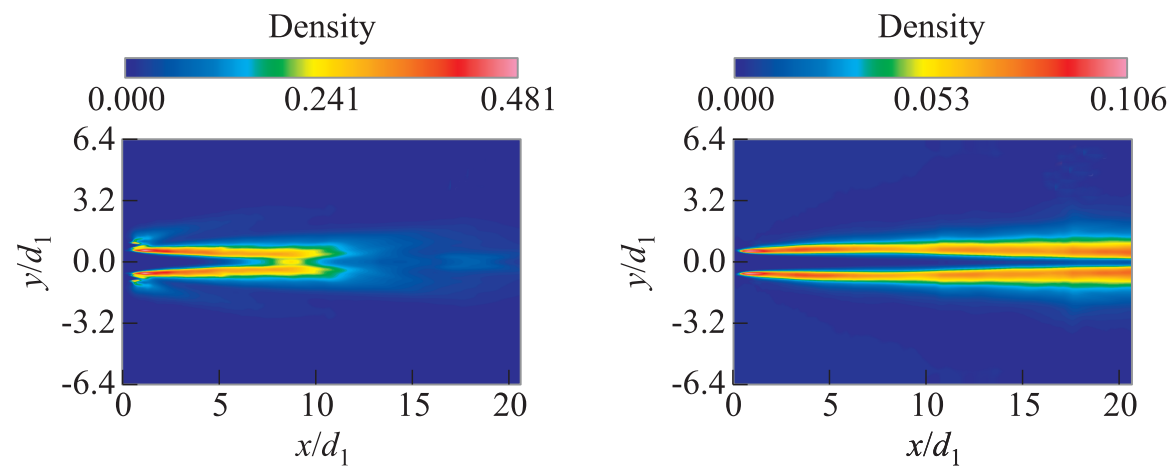

(a)
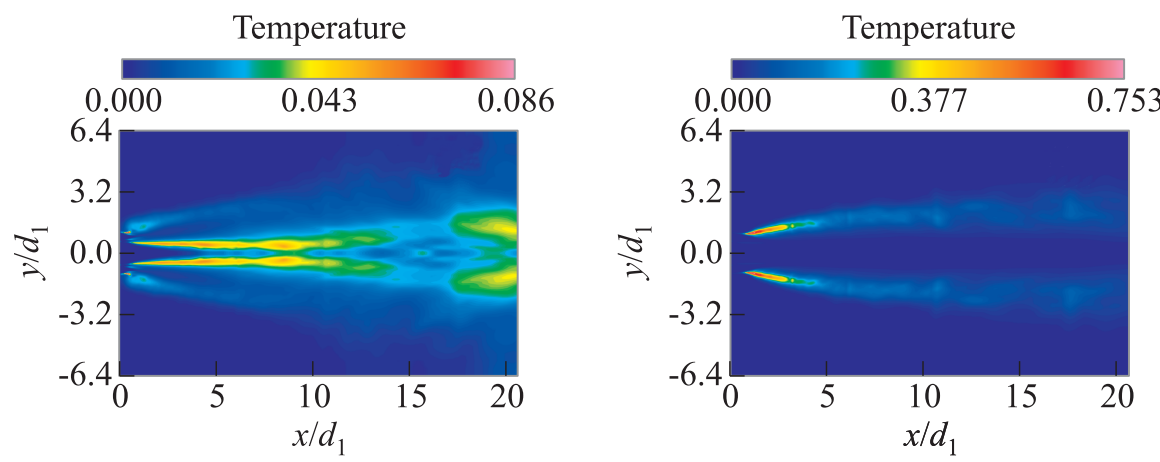

(b)

Figure 10 Fluctuation distributions scaled by the mean distributions for Cases 1 (left column) and 2 (right column): (a) density; and (b) temperature 
interacts with the chamber fluid) is relatively negligible. Similar to Case 1, the density fluctuation in Case 2 is generated primarily at the region between the inner and outer jets, but its magnitude is about one-fifth that of Case 2, indicating a less unsteady shear layer.

The temperature distribution in Case 1 shows a small fluctuation, even at the jet interaction region where the large density fluctuation is observed. This is because, although the inner $\mathrm{N}_{2}$ jet at $97 \mathrm{~K}$ expands with the interaction of the warmer outer jet at $462 \mathrm{~K}$ after the injection, the temperature variation is limited as compared to the density variation in the transcritical regime, as shown in Fig. $9 a$ (e.g., see the range of 100 to $200 \mathrm{~K}$ ). Therefore, as also stated in [11], small temperature fluctuations lead to large density fluctuations and vice versa owing to the strong variation in thermodynamics in this flow regime. On the other hand, quite large temperature fluctuations occur in Case 2 at the shear layer between the outer $\mathrm{H}_{2}$ jet at $52 \mathrm{~K}$ and the chamber $\mathrm{N}_{2}$ fluid at $810 \mathrm{~K}$. This may be simply due to the large temperature difference of $\Delta T \approx 750 \mathrm{~K}$ between the two fluids, which is quite different from $\Delta T \approx 45 \mathrm{~K}$ between the inner and outer jets or those of Case 1.

\subsection{Comparison of Dense-Core Length}

Figure 11 shows a comparison of dense-core length for experimental and computational data selected from earlier studies for coaxial injectors under supercritical pressures. The dense-core length is scaled with an inner diameter of $d_{1}$ $=2.0 \mathrm{~mm}$ and the momentum flux ratio is defined as $J$ $=\left(\rho u^{2}\right)_{o} /\left(\rho u^{2}\right)_{i}$. Note that the injector geometry and working fluid are not the same among the data.

The experimental plot from Davis and Chehroudi [4] represents the data under near-

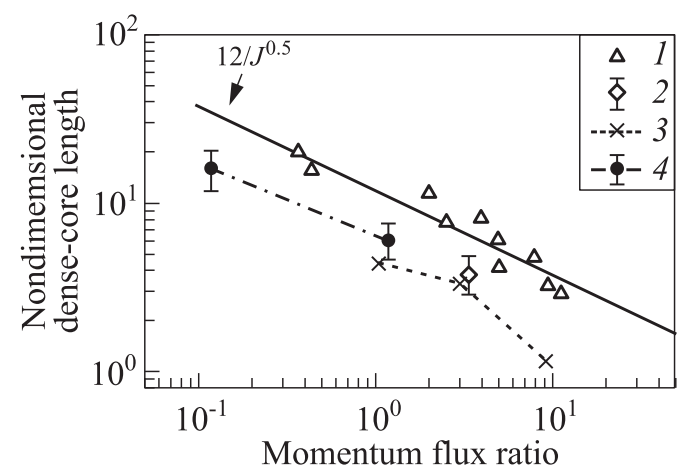

Figure 11 Comparison of nondimensional densecore length in terms of momentum flux ratio of outer jet to inner jet with earlier data: $1-\mathrm{N}_{2} / \mathrm{N}_{2}$, experiment [4]; $2-\mathrm{N}_{2} / \mathrm{H}_{2}$, experiment [3]; $3-$ $\mathrm{N}_{2} / \mathrm{N}_{2}$, computations [13]; and $4-\mathrm{N}_{2} / \mathrm{H}_{2}$, present study critical and supercritical conditions, in which they also provide the data under subcritical pressures. The experimental value from Oschwald et al. [3] is recalculated with Eq. (6) using the range of $0.7<\rho^{*}<0.9$. The computational data from Schmitt et al. [13] show a characteristic dense-core length, which is estimated by a position where the density takes a value of $\rho=0.5\left(\rho_{i}-\rho_{o}\right)+\rho_{o}$, which is somewhat different from 
Eq. (6) and, moreover, it is also noted that their injector had a recess. Because the definition of the dense-core region is not clearly made either in the experiments or in the computations, the present dense-core length is determined with an assumption that the dense core corresponds to the nondimensional densities of $0.7<\rho^{*}<0.9$.

With Fig. 11, it is found that the present dense-core length follows the slope of $J^{-0.5}$. The computational data of Schmitt et al. [13] generate a similar slope in a different range of momentum flux ratios. Thus, the combined computational data confirm the dense-core length to be $J^{-0.5}$, which is consistent with earlier single-phase experiments of coaxial jets, such as a gas-gas experiment [26]. The experimental data from Oschwald et al. [3] seem to be placed on the extended line of the present results and is almost identical to the results of Schmitt et al. Although there is a difference of the constant for the slope between the data of Davis and Chehroudi $12 J^{-0.5}$ and other data $\left(\sim(7-8) J^{-0.5}\right)$, the present results, covering small momentum flux ratios, support the claim that the densecore length is $J^{-0.5}$ for a wide range of momentum flux ratios. The constant for the slope may depend on inlet conditions such as velocity profile or perturbation level. The present results may also indicate the independence of mixture ratio on determining the dense-core length, considering the other experimental and computational data.

\section{CONCLUDING REMARKS}

A 3D numerical simulation of $\mathrm{N}_{2} / \mathrm{H}_{2}$ mixing for a coaxial injector under a supercritical pressure of $10 \mathrm{MPa}$ was carried out using a high-order numerical method. The effect of outer jet temperature on jet mixing was investigated under a constant mixture ratio and coaxial injector dimensions. The two outer $\mathrm{H}_{2}$ jet with temperatures of approximately 52 (Case 2) and $462 \mathrm{~K}$ (Case 1) were injected, whereas the inner $\mathrm{N}_{2}$ jet with an injection temperature of approximately $97 \mathrm{~K}$ is applied. In addition, a single-jet simulation (Case 3) was performed in order to examine the effect of the outer jet on jet mixing.

Overall, the outer jet with higher temperature and, therefore, larger momentum flux ratio (Case 1) generated a highly unsteady flow field because of the higher velocity shear between the inner and outer jets, resulting in better mixing field and a shorter dense-core region of the inner jet. In contrast, the outer jet with lower temperature and, therefore, smaller momentum flux ratio (Case 2) provided some stabilization of the inner jet, producing a relatively long densecore region of the inner jet, which was more pronounced in comparison with the single-jet case (Case 3), where interestingly the single jet had a shorter densecore length than that in Case 2. In Case 2, owing to the stabilization effect and the thermodynamic characteristics in the transcritical transition, the change in temperature of the inner jet on the centerline was minimal, creating a flattened 
region of temperature, which was quite different from the results for Case 1 . The present result of instantaneous and mean flow fields demonstrates that even if the same mixture ratio is set for a axial injector, the resultant mixing fields are very different.

The fluctuation flow fields show that the large density fluctuation mainly occurs at the shear layer between the inner and outer jets for Cases 1 and 2. On the other hand, in Case 1, the temperature fluctuation is observed at the same shear layer but with a smaller magnitude owing to the thermodynamic variation in the transcritical regime. The temperature fluctuation in Case 2, in contrast, occurs at different shear layers between the outer jet and the chamber fluid owing to the significant difference in temperature.

Finally, through the comparison of dense-core length with earlier experimental and computational data, the present results confirmed the fact that the dense-core length is proportional to $J^{-0.5}$ (where $J$ is the momentum flux ratio) for a wide range of $J$ values, whereas there is still uncertainty in terms of determining the coefficient of the slope.

Further investigation, e.g., a comparison while keeping not only the mixture ratio but also the momentum flux ratio constant using different injector dimensions, is now underway.

\section{REFERENCES}

1. Yang, V., M. Habiballah, J. Hulka, and M. Popp. 2004. Liquid rocket thrust chambers: Aspects of modeling, analysis, and design. Progress in astronautics and aeronautics ser. Reston, VA, USA: AIAA. Vol. 200. 743 p.

2. Mayer, W., and H. Tamura. 1996. Propellant injection in a liquid oxygen/gaseous hydrogen rocket engine. J. Propul. Power 12(6):1137-1147.

3. Oschwald, M., A. Schik, M. Klar, and W. Mayer. 1999. Investigation of coaxial $\mathrm{LN}_{2} / \mathrm{GH}_{2}$-injection at supercritical pressure by spontaneous Raman scattering. AIAA Paper No. 99-2887.

4. Davis, D. W., and B. Chehroudi. 2007. Measurements in an acoustically driven coaxial jet under sub-, near-, and supercritical conditions. J. Propul. Power 23(2):364-374.

5. Locke, J. M., S. Pal, R. D. Woodward, and R. J. Santoro. 2010. High speed visualization of $\mathrm{LOx} / \mathrm{GH}_{2}$ rocket injector flowfield: Hot-fire and cold-flow experiments. AIAA Paper No. 2010-7145.

6. Zong, N., and V. Yang. 2006. Cryogenic fluid jets and mixing layers in transcritical and supercritical environments. Combust. Sci. Technol. 178(1):193-227.

7. Schmitt, T., L. Selle, A. Ruiz, and B. Cuenot. 2010. Large-eddy simulation of supercritical-pressure round jets. AIAA J. 48(9):2133-2144.

8. Mayer, W., J. Telaar, R. Branam, G. Schneider, and J. Hussong. 2003. Raman measurements of cryogenic injection at supercritical pressure. Heat Mass Transfer 39(8):709-719. 
9. Park, T. 2012. LES and RANS simulations of cryogenic liquid nitrogen jets. J. Supercrit. Fluid. 72:232-247.

10. Terashima, H., and M. Koshi. 2013. Unique characteristics of cryogenic nitrogen jets under supercritical pressures. J. Propul. Power 29(6):1328-1336.

11. Hosangadi, A., C. Lee, C. Kannepalli, and S. Arunajatesan. 2008. Threedimensional hybrid RANS/LES simulations of a supercritical liquid nitrogen jet. AIAA Paper No. 2008-5227.

12. Masquelet, M., N. Guezennec, and S. Menon. 2012. Numerical studies of mixing and flame-turbulence interactions in shear coaxial injector flows under trans-critical conditions. AIAA Paper No. 2012-1269.

13. Schmitt, T., J. Rodriguez, I. Leyva, and S. Candel. 2012. Experiments and numerical simulation of mixing under supercritical conditions. Phys. Fluids 24:055104.

14. Terashima, H., and M. Koshi. 2012. Approach for simulating gas-liquid-like flows under supercritical pressures using a high-order central differencing scheme. J. Comput. Phys. 231(20):6907-6923.

15. Soave, G. 1972. Equilibrium constants from a modified redlich-kwong equation of state. Chem. Eng. Sci. 27(6):1197-1203.

16. Chung, T., M. Ajlan, L. Lee, and K. Starling. 1988. Generalized multiparameter correlation for nonpolar and polar fluid transport properties. Ind. Eng. Chem. Res. 27(4):671-679.

17. Riazi, M., and C. Whitson. 1993. Estimating diffusion coefficients of dense fluids. Ind. Eng. Chem. Res. 32(12):3081-3088.

18. Cook, A., and W. Cabot. 2004. A high-wavenumber viscosity for high-resolution numerical methods. J. Comput. Phys. 195(2):594-601.

19. Cook, A. 2007. Artificial fluid properties for large-eddy simulation of compressible turbulent mixing. Phys. Fluids 19:055103.

20. Kawai, S., and S. Lele. 2008. Localized artificial diffusivity scheme for discontinuity capturing on curvilinear meshes. J. Comput. Phys. 227(22):9498-9526.

21. Lele, S. 1992. Compact finite difference schemes with spectral-like resolution. J. Comput. Phys. 103(1):16-42.

22. Gottlieb, S., and C. Shu. 1998. Total variation diminishing Runge-Kutta schemes. Math. Computation 67(221):73-85.

23. Gaitonde, D., and M. Visbal. 2000. Padé-type higher-order boundary filters for the Navier-Stokes equations. AIAA J. 38(11):2103-2112.

24. Terashima, H., S. Kawai, and N. Yamanishi. 2011. High-resolution numerical method for supercritical flows with large density variations. AIAA J. 49(12):26582672 .

25. Lemmon, E., M. Huber, and M. McLinden. 2007. NIST Standard Reference Database 23: Reference Fluid Thermodynamic and Transport PropertiesREFPROP, Version 8.0. National Institute of Standards and Technology, Standard Reference Data Program, Gaithersburg, MD.

26. Favre-Marinet, M., and E. Camano Schettini. 2001. The density field of coaxial jets with large velocity ratio and large density differences. Int. J. Heat Mass Transfer 44(10):1913-1924. 